\title{
Fronteira de Disparidade Regional
}

\author{
Regional Inequality Frontier
}

Leonardo Monasterio*

Resumo: Os conceitos de fronteira de disparidade regional e razão de desigualdade regional são apresentados no texto. Milanovic, Lindert e Williamson (2011) criaram o conceito de inequality possibility frontier. Eles constataram que sociedades muito pobres não podem exibir elevados índices de Gini de desigualdade de renda porque o excedente econômico é pequeno demais para ser apropriado pelos estratos superiores. A inequality possibility frontier é formada pelo nível máximo de desigualdade que pode ser atingido a cada nível de renda. Este estudo estende tais conceitos para tratar da dimensão regional. Países com níveis de renda próximos da subsistência têm de apresentar baixos níveis de desigualdade regional de renda per capita. Os novos conceitos para a avaliação das disparidades regionais em longo prazo são aplicados à experiência brasileira entre 1872 e 2008.

Palavras-chave: Desigualdade regional. Indicadores de desigualdade. Índice de Gini.

Abstract: The concepts of regional inequality frontier and regional inequality ratio are presented in this paper. Milanovic, Lindert and Williamson (2011) introduced the concept of the inequality possibility frontier. Their starting point is that very poor societies will never display high Gini indexes of personal distribution of income because there is very little surplus to be appropriated by the upper classes of these societies. The inequality possibility frontier is the maximum level of inequality possible at each level of income. This paper extends the concept to cover regional cases. Countries with populations close to subsistence level inevitably display low regional inequality of income per capita. Rising levels of wealth imply higher attainable degrees of regional inequality. The Brazilian experience between 1872 and 2008 provide an opportunity to apply these new concepts for appraising regional inequality in the long term.

Keywords: Regional inequality. Inequality index. Gini index.

JEL Classification: R10; N96.

Doutor em Desenvolvimento Econômico (UFPR). Professor do Programa de Pós-graduação em Economia da Universidade Católica de Brasília (UCB). Técnico de Planejamento e Pesquisa do Ipea. E-mail: leonardo.monasterio@gmail.com

Sou grato à Fundación Carolina e ao programa de doutorado em História Econômica da Universidade Carlos III de Madrid por proporcionarem as condições para a finalização do artigo. Agradeço também aos pareceristas anônimos pelas correções e sugestões. Os erros remanescentes são meus. 


\section{Introdução}

O presente texto traz uma contribuição metodológica para o estudo da desigualdade regional em longo prazo. O ponto de partida para a sua apresentação é o artigo clássico de Jeffrey Williamson (1965). No estudo, o autor identificou a existência de uma curva em $U$ invertido que relacionaria desigualdade regional e renda per capita. ${ }^{1}$ As disparidades espaciais seriam pouco expressivas em países muito pobres. Já nos momentos iniciais do desenvolvimento econômico, algumas regiões do país atrairiam capitais, migrantes mais bem qualificados, ou mesmo, maiores investimentos públicos. Seria um mundo à moda de Myrdal (1963), em que forças centrípetas levam a um aumento da desigualdade entre as regiões. A partir de certo ponto, os mecanismos clássicos de movimentos de capital e trabalho (e, em alguns casos, as políticas públicas) conduziriam à queda da desigualdade de renda per capita entre as regiões. ${ }^{2}$

Mais de 40 anos após a publicação de seu artigo sobre desigualdade regional, Williamson, junto com Milanovic e Lindert, desenvolveram os conceitos de fronteira de possibilidade de desigualdade (inequality possibility frontier) e razão de desigualdade de extração (inequality extraction ratio) (MILANOVIC; LINDERT; WILLIAMSON, 2011). ${ }^{3}$ Elaborados para a análise da evolução da distribuição pessoal de renda, esses conceitos incorporam a noção de que existem limites máximos - a cada nível de renda - para a desigualdade.

Este trabalho mostra que o insight obtido por Milanovic, Lindert e Williamson (2011) - com as adaptações necessárias - pode ser aplicado à análise da desigualdade regional em longo prazo. Chega-se, assim, aos conceitos de fronteira de disparidade regional e razão de desigualdade regional.

A fronteira de disparidade regional é formada pela máxima desigualdade entre unidades espaciais possível de ser observada para um dado nível de renda per capita em um país. Já a razão de desigualdade regional é a relação entre a desigualdade regional medida e a dada pela fronteira de desigualdade. Os dois conceitos são a contribuição metodológica a que se propõe o artigo.

A próxima seção sintetiza as duas contribuições de Milanovic, Lindert e Williamson (2011): inequality possibility frontier e inequality extraction ratio. A terceira seção, por sua vez, apresenta os conceitos de fronteira de disparidade

$1 \quad$ Vale lembrar que, apesar do U invertido de Williamson ter paralelo na curva de mesmo formato identificada por Kuznets (1955), uma trata da desigualdade regional e outra da pessoal. Tratam-se de aspectos analítica e empiricamente distintos, e há que se ter cuidado na distinção entre ambas ao longo do presente texto.

2 Barrios e Strobl (2009) não refutaram a hipótese do U invertido para dados internacionais recentes e com técnicas econométricas sofisticadas.

3 Na verdade, o conceito de inequality possibility frontier já havia sido apresentado em Milanovic (2006). 
regional razão de desigualdade regional para os casos dos índices de Gini e para o índice Vw. Na quarta seção, a fronteira de desigualdade e a razão de desigualdade regional são calculadas para os estados brasileiros no período 1872-2008. Para encerrar, a quinta seção apresenta as considerações finais do trabalho.

\section{Fronteira de Possibilidade de Desigualdade}

A melhor forma de se apresentar a contribuição de Milanovic, Lindert e Williamson (2011) é por meio de um simples exemplo. Suponha-se uma sociedade com 100 habitantes que produzem o equivalente a 110 salários de subsistência. 90 desses indivíduos sobrevivem com apenas um salário de subsistência, enquanto 10 ganham o restante disponível, ou seja, o equivalente a 20 salários de subsistência. O índice de Gini dessa sociedade não está próximo da unidade, mas sim de 0,08 . Portanto, o índice de Gini sugere uma sociedade igualitária, porém, na verdade, todo o excedente é apropriado por um pequeno grupo. Milanovic, Lindert e Williamson (2011) perceberam que, em níveis de renda per capita muito baixos, o índice de Gini é fatalmente baixo por uma restrição matemática.

A inequality possibility frontier mostra o índice de Gini possível para cada nível de renda per capita em uma sociedade (MILANOVIC; LINDERT; WILLIAMSON, 2011). No caso extremo, ter-se-ia uma sociedade com duas classes de indivíduos: a parcela $\varepsilon$ da população faz parte da elite e (1- $\varepsilon$ ) que compõe o restante da sociedade. O ponto de partida da inequality possibility frontier é o cálculo da renda máxima possível $\left(y_{h}\right)$ desse grupo pequeno de indivíduos da classe de renda alta.

Seja:

a) $s$ = renda de subsistência definida em termos biológicos;

b) $N$ = número de pessoas na sociedade;

c) $\mu=$ renda per capita da sociedade como um todo;

d) $\varepsilon=$ número de pessoas na elite/número de pessoas na sociedade.

Logo:

$$
\begin{aligned}
& y_{h}=\frac{\mu N-s N(1-\varepsilon)}{\varepsilon N} \\
& \left.y_{h}=\frac{1}{\varepsilon}[\mu-s(1-\varepsilon)]\right]
\end{aligned}
$$


O índice de Gini para indivíduos que estão ordenados em classes ascendentes de renda é simplificado quando se supõe que haja plena igualdade dentro das classes e que não haja sobreposição entre as classes. Ou seja, ninguém da classe inferior aufere renda maior do que qualquer um da classe superior. Dessa forma,

$$
G=\frac{1}{\mu}\left(y_{j}-y_{i}\right) p_{j} p_{i}
$$

em que pi e pj representam a parcela da população nas classes $i$ e $j\left(y_{j}>y_{i}\right)$. Fazendo-se $y_{h}=y_{j}, y_{i}=s, \varepsilon=p_{j} e(1-\varepsilon)=p_{i}$, pode-se chegar ao cálculo do índice máximo de Gini $\left(G^{\prime}\right)$ :

$$
G^{\prime}=\frac{1-\varepsilon}{\mu}(\mu-s)
$$

Para que se tenha a renda per capita como múltiplo da renda de subsistência, faz-se $\mu=\alpha$ s. Ou seja, $\alpha$ representa quantas vezes a renda per capita de uma economia supera a de subsistência biológica.

$$
G^{\prime}=\frac{\alpha-1}{\alpha}(1-\varepsilon)
$$

Com $\varepsilon=0,10$, os valores de G' estão de acordo com a Tabela 1. Ou seja, para um país em que a renda per capita seja o dobro da de subsistência $(\alpha=2)$, no qual haja uma elite composta por $10 \%$ da população, o maior índice de Gini possível seria igual a 0,45 . Já um país com $\alpha=4$ terá um Gini máximo ( $G$ ') de 0,68 . Obviamente, conforme a renda per capita média se afasta da de subsistência, valores de Gini próximos à 1 são possíveis.

Tabela 1 - Simulação do índice de Gini máximo (G') de acordo com os múltiplos do nível de renda per capita de subsistência $(\alpha)$

\begin{tabular}{cc}
\hline$\alpha$ & $\mathrm{G}^{\prime}$ \\
\hline 1 & 0,00 \\
1,5 & 0,30 \\
2 & 0,45 \\
2,5 & 0,54 \\
3 & 0,60 \\
3,5 & 0,64 \\
4 & 0,68 \\
4,5 & 0,70 \\
5 & 0,72 \\
\hline
\end{tabular}

Fonte: Cálculos do autor a partir de Milanovic, Lindert e Williamson (2011). 
A inequality possibility frontier proposta por Milanovic, Lindert e Williamson (2011) é construída diretamente a partir da equação 5. Finalmente, os autores propuseram o conceito de inequality extraction ratio, ou seja, a razão entre o Gini observado e o G'. Valores próximos da unidade significam que quase todo o excedente disponível é apropriado pela elite. No caso da sociedade exemplificada na introdução, a inequality extraction ratio seria igual a um, indicando o quão desigual de fato é. ${ }^{4}$

\section{Fronteira de Disparidade Regional}

A mesma questão apontada por Milanovic, Lindert e Williamson (2011) no tocante à distribuição pessoal da renda surge na análise da desigualdade regional. As rendas per capita das regiões não podem ser muito dispersas em níveis baixos de renda porque há o limite inferior e o excedente não é grande o suficiente para permitir que as regiões mais ricas se distanciem.

A transposição da inequality possibility frontier para o âmbito regional é feita de forma bastante direta. Ao invés de se analisarem famílias ou classes sociais, o foco volta-se para as unidades espaciais. Conforme já foi dito, para construir a inequality possibility frontier, Milanovic, Lindert e Williamson (2011) criam um contrafatual que atribui aos indivíduos da classe superior todo o excedente produzido, enquanto os demais permanecem na subsistência. Essa elite repartiria igualmente a renda extraída. No caso regional, o contrafatual é construído de forma semelhante: para cada nível de renda, atribui-se a uma unidade (no caso, um estado) toda a renda excedente produzida na economia.

\subsection{Fronteira de Disparidade Regional (Gini)}

O Gini máximo regional $\left(\mathrm{G}^{*}\right)$ é obtido da mesma forma proposta para o cálculo de Gini máximo para a distribuição pessoal da renda (G'), porém nesse caso $\varepsilon$ representa a participação da unidade mais rica do país (ou área de referência) na população como um todo. ${ }^{5}$ Enquanto Milanovic, Lindert e Williamson (2011) tiveram que escolher certo valor percentual que representasse o papel da elite na sociedade, o dever aqui é arbitrar a unidade mais rica quando se simula uma concentração espacial máxima da renda. O candidato natural é aquele local (no caso, o estado) que já é - de fato - o mais rico. Calcula-se, assim, sua renda per capita supondo-se que os demais estão no nível de subsistência, e transfere-se todo

$4 \quad$ Milanovic, Lindert e Williamson mostraram duas propriedades desejáveis da inequality possibility frontier: não há grande variação nos G' em relação a mudanças razoáveis no $\varepsilon$. O relaxamento da hipótese de que toda a elite ganha a mesma renda não altera em demasiado os resultados.

5 É prudente notar que todos os indicadores regionais aqui apresentados estão sujeitos ao conhecido problema do modifiable areal unit problem. Ver Haining (2003). 
o excedente para a tal unidade mais rica. Dessa maneira, a parcela $\varepsilon$ não mais corresponde à parcela da elite, mas sim à participação da população que vive na unidade mais rica do país. No mais, o cálculo de $G^{*}$ é idêntico ao apresentado na equação 5 .

\subsection{Fronteira de Disparidade Regional ( $V w$, de Williamson)}

O índice Vw nada mais é que um coeficiente de variação da renda per capita ponderado pela parcela da população em cada região. Em sua versão original, é calculado da seguinte forma (WILLIAMSON, 1965):

$$
V_{w}=\frac{\sqrt{\sum\left(y_{i}-\mu\right)^{2}\left(p_{i} / N\right)}}{\mu}
$$

em que: $\mu=$ renda per capita do país; $y_{i}=$ renda per capita da unidade espacial $i ; p_{i}=$ população da unidade espacial $i ; N=$ população do país;

Como é um coeficiente de variação, os limites teóricos do índice de Williamson são $[0, \infty)$. Para o cálculo do valor máximo do índice de Williamson $\left(V_{w}^{*}\right)$, cria-se um contrafatual semelhante ao utilizado no caso do $G^{*}$ : a população de todas as unidades aufere renda de subsistência, exceto à da unidade líder. Esta reparte todo o excedente. A equação 6 fica reduzida, então, a

$$
V_{w} *=\frac{\sqrt{(s-\mu)^{2}(1-\varepsilon)}+\sqrt{\left(y_{h}-\mu\right)^{2} \varepsilon}}{\mu}
$$

Além da variável $\mu$ definida na equação 6, tem-se: $s=$ renda de subsistência biológica; $\mu$ = renda per capita do país; $\varepsilon=$ parcela da população na unidade mais rica. 
Como foi mostrado, yh pode ser calculado com base na equação 1. Simulando-se $V_{w}{ }^{*}$ para diversos valores de $\alpha$ e $\varepsilon=0,1$, tem-se a Tabela 2 .

Tabela 2 - Simulação do índice de Williamson máximo $\left(V_{w}^{*}\right)$ de acordo com os múltiplos do nível de renda per capita de subsistência $(\alpha)$

\begin{tabular}{cc}
\hline$\alpha$ & $\mathrm{V}_{\mathrm{w}}{ }^{*}$ \\
\hline 1 & 0,00 \\
1,5 & 1,26 \\
2 & 1,90 \\
2,5 & 2,28 \\
3 & 2,53 \\
3,5 & 2,71 \\
4 & 2,85 \\
4,5 & 2,95 \\
5 & 3,04 \\
\hline Fonte: Elaboração própria.
\end{tabular}

\subsection{Razão de Desigualdade Regional}

Analogamente ao que ocorre com a inequality extraction ratio, a razão de desigualdade regional é dada pela divisão do indicador de desigualdade observado pelo máximo para cada nível de renda. Assim, nos casos dos índices de Gini e $V_{w}$, tem-se:

$$
\begin{gathered}
R D R_{V w}=\frac{V w}{V w^{*}} \\
R D R_{G}=\frac{G}{G^{*}}
\end{gathered}
$$

Uma razão de desigualdade regional próxima da unidade indica que a desigualdade observada é próxima da maior possível. Vale notar que a trajetória da razão de desigualdade regional ao longo do processo de desenvolvimento não é trivial. Basta que a disparidade regional observada cresça em ritmo maior que o do limite máximo dos indicadores de desigualdade para que a razão de desigualdade regional aumente. 


\section{Fronteira de Disparidade Regional e Razão de Desigualdade Regional no Caso Brasileiro entre 1872 e 2008}

Não é o objetivo do texto discutir as raízes, nem as razões subjacentes às trajetórias regionais no Brasil, mas vale a pena apresentar um pouco do contexto. É muito importante lembrar que, como um todo, o Brasil era muito pobre em 1872. Sua renda per capita era de apenas 1,8 vez a de subsistência, e um terço da observada no Uruguai no mesmo ano (MADDISON, 2010).

Também se sabe que o Brasil já tinha marcadas disparidades regionais em 1872: o atraso relativo do nordeste estava consolidado e a ascensão do centro-sul, clara. Por sua vez, no século XX, a rápida industrialização costuma ser responsabilizada pela ampliação e manutenção da desigualdade regional no Brasil. Williamson (1965) afirma que o caso brasileiro se encaixaria bem na trajetória de $U$ invertido no período de 1939 a 1959. A desigualdade teria alcançado o máximo em 1952 - um recorde internacional na época - e caído desde então. Azzoni (1997) mostrou que, em linhas gerais, a desigualdade entre os estados seguiu caindo nas décadas seguintes, estabilizando-se após 1985. Em períodos ainda mais recentes, uma miríade de estudos de convergência de renda per capita mostra resultados não tão consensuais sobre a tendência da desigualdade regional entre os estados. ${ }^{6}$

\subsection{Procedimentos}

Neste trabalho, as rendas per capita foram calculadas partindo-se da participação dos estados no PIB brasileiro. Elas foram obtidas nas seguintes fontes:

a) de Bértola, Castelnovo e Willebald (2009) para 1872;

b) para o PIB de 1920, os dados do PIB municipal calculados por Eustáquio Reis foram agregados nos estados. As informações estão disponíveis no Ipeadata (INSTITUTO DE PESQUISA ECONÔMICA APLICADA, 2010);

6 A literatura sobre convergência no Brasil é longa demais para ser resenhada aqui. Desde Ferreira e Diniz (1995) até Maia (2010), passando por Mossi et al. (2003), o caso brasileiro é um dos mais estudados, com métodos e bases de dados bastante diversos. Recentemente, Rezende (2011) mostrou como os resultados de convergência brasileiros para dados mais recentes são susceptíveis à mudança do nível de agregação espacial.

7 Buescu (1979) estimou as rendas per capita provinciais de 1872 com base em dados de exportações. Contudo, o grau da disparidade regional parece exagerado, pois há províncias com rendas per capita mais de 30 vezes maiores que outras. Reis (2009) calculou as rendas per capita estaduais das áreas mínimas comparáveis e obteve resultados estaduais não muito distantes dos de Bértola, Castelnovo e Willebald (2009). O coeficiente de correlação entre as duas séries é de 0,9. A série de Reis (2009) não foi utilizada porque em seu trabalho Amazonas e Mato Grosso tiveram de ser agregados na construção das áreas mínimas comparáveis no período 1872-2000 (ver Reis, Pimentel e Alvarenga, 2007). 
c) para os anos seguintes, a fonte é o Ipeadata (INSTITUTO DE PESQUISA ECONÔMICA APLICADA, 2010), que por sua vez está baseada nas estimativas feitas pela Fundação Getulio Vargas para os anos iniciais e pelo Instituto Brasileiro de Geografia e Estatística (IBGE) para o período de 1970 em diante.

Feitas as agregações necessárias entre os estados ${ }^{8}$ e tomando-se por base o PIB do Brasil estimado por Maddison (2010), ${ }^{9}$ chegou-se aos PIBs estaduais. Para o valor da renda per capita de subsistência, utilizaram-se US\$ 400 anuais, o mesmo limite escolhido por Maddison (2010).

A definição de uma renda per capita de subsistência é controversa. Até os limites de subsistência biológica podem variar no espaço. Colin Clark já apontava a necessidade de considerar unidades monetárias “orientais" (indianas) para efetuar comparações internacionais, uma vez que as necessidades de vestuário e alimentação variam de acordo com as condições climáticas (CLARK, 1957; MADDISON, 2004). Milanovic, Lindert e Williamson (2011) discutem as várias estimativas da renda per capita de subsistência e se decidem por US\$ 300.

Aqui, manteve-se o valor de US\$ 400 dólares (poder paridade de compra) de 1990 para que fosse mantida a consistência com a estimativa de Maddison para a renda per capita brasileira. ${ }^{10}$

Esse procedimento resultou em rendas per capita para alguns estados do nordeste brasileiro menores que a de subsistência. Ou seja, a estimativa de renda per capita estadual em 1920 (INSTITUTO DE PESQUISA ECONÔMICA APLICADA, 2010), em conjunto com a de Maddison (2010) para o Brasil, resultaria em populações com renda per capita nesse ano inferior à subsistência. É fato que as secas de 1915 no nordeste puseram milhares de habitantes abaixo da linha de subsistência. Contudo, é mais provável que tal discrepância seja resultante de: a) métodos de cálculo da cesta de subsistência e do câmbio PPP; e b) diferenças regionais nas necessidades biológicas de sobrevivência. A série foi então ajustada para que o estado mais pobre (o Ceará de 1920, com US\$ 315) tivesse uma renda per capita de US\$ 400 (ver Anexo). Vale notar que mudanças nesse valor de subsistência não alteram qualitativamente os resultados do artigo. ${ }^{11}$

8 Quando apropriado, o Mato Grosso do Sul foi agregado ao Mato Grosso; Acre, Rondônia e Roraima, ao Amazonas; e Tocantins e o Distrito Federal, a Goiás.

9 Em dólares Geary-Khamis com preços de 1990, cujo valor leva em consideração a paridade do poder de compra (PPP) entre as moedas.

10 Milanovic (2006) e MLW (2011, nota 17) discutem a construção da estimativa de renda per capita de subsistência fisiológica. Gregory Clark (2009) critica o valor de US\$ 400 escolhido por Maddison e a excessiva generosidade com que ele imputou tal nível de renda aos países do passado.

11 Os resultados utilizando outros níveis de renda per capita de subsistência podem ser solicitados ao autor. 
O estado do Rio de Janeiro foi o de maior renda per capita até 1950. Entretanto, em 1960, São Paulo aparece à sua frente e mantém a liderança até o fim do período. Essa mudança impõe decidir se será mantido o $\varepsilon$ referente ao Rio de Janeiro ou se utilizado o de São Paulo ao longo da série. Optou-se por preservar o $\varepsilon$ fluminense em cada ano, até o fim do período. ${ }^{12} \mathrm{O}$ exercício foi repetido com São Paulo como estado de referência e os resultados não foram substancialmente alterados.

\subsection{A Evolução da Desigualdade Regional no Brasil}

O Gráfico 1 apresenta a evolução dos índices de Gini (ponderados pela população) e de $V_{w}$ para toda a série.

Gráfico 1 - Índice de Gini e Vw para o PIB per capita dos estados brasileiros (1872-2008)

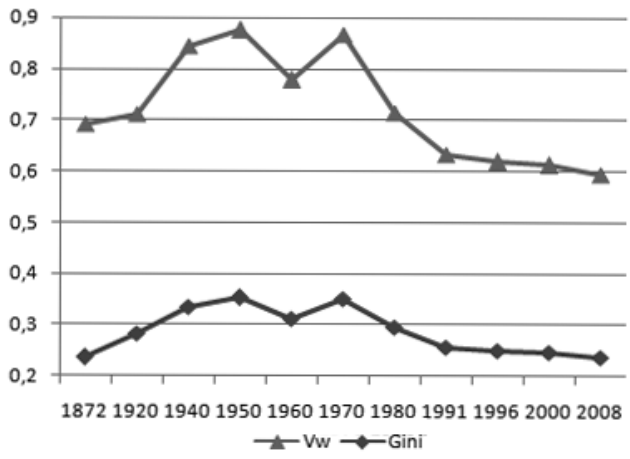

Fonte: Elaboração própria.

Como era de se esperar, as curvas de Vw e Gini apresentam os seguintes resultados: a desigualdade entre estados aumenta até meados do século XX, caindo a partir de então. De grosso modo, o $U$ invertido de Williamson seria válido. Apenas o período de 1960-1970 escapa desse padrão, visto a desigualdade crescente. ${ }^{13}$

12 São Paulo passou da condição de estado com uma renda per capita próxima da brasileira em 1872 (com 37\%) para a de industrial powerhouse brasileira no século XX. Nesse decênio do século XIX, o café paulista era responsável por apenas $11 \%$ da produção nacional e possuía $6 \%$ dos imigrantes. Nas décadas seguintes deu-se a expansão cafeeira no oeste paulista, viabilizadas pela ferrovia e pela mão de obra imigrante. Ao longo da década de 1910, São Paulo já produzia mais de dois terços do café brasileiro, possuía $60 \%$ dos imigrantes e um terço dos estabelecimentos industriais brasileiros. Ver Reis e Monasterio (2010) para uma análise quantitativa das raízes desse fenômeno. Cano (1998) é a referência clássica.

13 A análise de Azzoni (1997) sugere que o aumento da desigualdade regional em 1970 decorreria da divergência observada nos últimos anos da década de 1960, que estaria associada, no caso brasileiro, aos momentos de rápido crescimento econômico. 


\subsection{Análise da Fronteira de Disparidade Regional e da Razão de Desigualdade Regional no Brasil}

O Gráfico 2 apresenta o $G, G^{*}$ e a razão de desigualdade regional baseada no índice de Gini $\left(R D R_{G}\right)$, mantendo-se o $\varepsilon$ referente à participação do Rio de Janeiro em cada ano de análise. $O G^{*}$ cresce por toda a série - a evolução do $G$ observado já foi comentada. O que chama atenção é o indicador da razão de desigualdade regional, que ficou praticamente estável e em patamar alto, por volta de 0,57 no período 1872-1920. Ou seja, apesar do baixo índice de Gini apresentado, era cerca de 57\% do máximo possível. A partir de 1920, a tendência é a queda na razão de desigualdade regional, salvo o "repique" em 1970. Quando a desigualdade regional mais cresceu, no início da decolagem (ROSTOW, 1959), a razão entre a desigualdade observada e a possível caiu. Isto é, apesar de se ter uma economia mais desigual, ela se afastou da desigualdade máxima potencial. Outra forma de se avaliar esse resultado seria dizer que a desigualdade no começo do período só não foi maior porque o Brasil era tão pobre que não poderia sustentá-la.

Gráfico 2 - Gini* e razão de desigualdade regional $\left(R D R_{\text {gini }}\right)$ para os estados brasileiros (1872-2008)

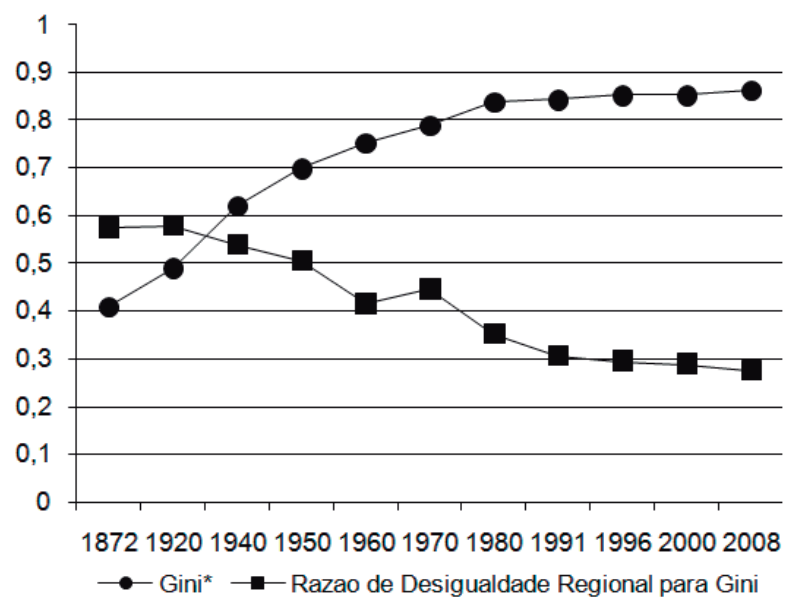

Fonte: Elaboração própria. 
Por sua vez, o Gráfico 3 apresenta os dados relativos ao $V_{w}, V_{w}$ " e à razão de desigualdade regional baseada em $V_{w}\left(R D R V_{w}\right)$. Os resultados são idênticos aos observados no caso do índice de Gini, salvo que os movimentos do índice $V_{w}$ são mais ampliados devido à sensibilidade maior do índice de Williamson aos valores extremos.

Gráfico $3-V_{w}{ }^{*}$ e razão de desigualdade regional $\left(R D R_{V w}\right)$ para os estados brasileiros (1872-2008)

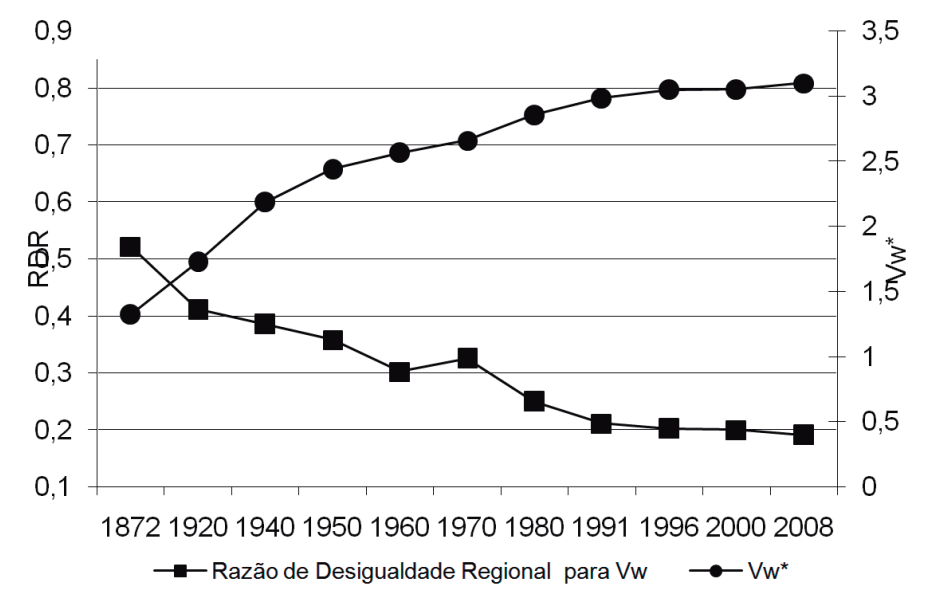

Fonte: Elaboração própria.

Nota: Os valores de $V_{w}{ }^{*}$ estão no eixo da direita.

\section{Considerações Finais}

Este trabalho contribuiu para o estudo das desigualdades regionais ao adaptar o conceito de inequality possibility frontier de Milanovic, Lindert e Williamson (2011) à dimensão regional. Evidenciou-se que parte da explicação para o trecho ascendente do $U$ invertido de Williamson (1965) decorre do simples fato de que níveis mais altos de desigualdade regional são inalcançáveis em países muito pobres. Mostrou-se, também, que o conhecido índice $V_{w}$ de Williamson está sujeito às mesmas distorções que caracterizam o índice de Gini regional para níveis de renda próximos aos de subsistência.

No Brasil de 1872, a economia estava mais próxima da desigualdade regional máxima possível que ao longo do século XX. Chama atenção a queda intensa da razão de desigualdade regional até 1950. No início do crescimento econômico moderno, quando a atividade econômica se concentrou no sudeste, o Brasil, na verdade, se afastava da fronteira de disparidade regional. Apesar de ser correta a visão de que as disparidades regionais aumentaram em meados do século XX, 
deve-se ter em mente que isso aconteceu porque havia um limite para uma desigualdade maior no início da série.

Vale reforçar o ponto principal: em estudos sobre a desigualdade regional em longo prazo, ou para países com níveis iniciais muito baixos de renda, recomenda-se o cálculo da fronteira de disparidade regional e da razão de desigualdade regional. As medidas tradicionais de desigualdade regional podem induzir interpretações equivocadas da evolução dessa dimensão.

\section{Referências}

AZZONI, C. Concentração regional e dispersão das rendas per capita estaduais: análise a partir de séries históricas estaduais de PIB, 1939-1995. Estudos Econômicos, v. 27, n. 2, p. 341-393, dez. 1997.

BARRIOS, S.; STROBL, E. The dynamics of regional inequalities. Regional Science and Urban Economics, v. 39, n. 5, p. 575-591, Sept. 2009.

BÉRTOLA, L.; CASTELNOVO, C.; WILLEBALD, H. Income distribution in Brazil, 1870-1920. Madrid: Instituto Figuerola, Universidad Carlos III, May 8-9, 2009. Disponível em: <http:// www.uc3m.es/portal/page/portal/instituto_figuerola/investigacion/programas/programa1/ conferencia/programme/BCWBrazilMadrid.pdf> . Acesso em: 31 mar. 2010.

BUESCU, M. Brasil: disparidades de renda no passado. Rio de Janeiro: Saraiva, 1979.

CANO, W. Raízes da concentração industrial em São Paulo. Campinas: Universidade Estadual de Campinas, 1998.

CLARK, C. The conditions of economic progress. London: Macmillan, 1957.

CLARK, G. Resenha de Contours of the World Economy, 1-2030 AD: Essays in MacroEconomic History de Angus Maddison. The Journal of Economic History, v. 69, n. 4, p. $1156-$ 1161, Dec. 2009.

FERREIRA, A.; DINIZ, C. C. Convergência entre as rendas per capita estaduais no Brasil. Revista de Economia Política, v. 15, n. 4, p. 38-56, 1995.

HAINING, R. Spatial data analysis: theory and practice. Cambridge: Cambridge University Press, 2003.

INSTITUTO DE PESQUISA ECONÔMICA APLICADA. Ipeadata. 2010. Disponível em:<http://www.ipeadata.gov.br/>. Acesso em: 31 mar. 2010.

KUZNETS, S. Economic growth and income inequality. The American Economic Review, v. 45, n. 1, p. 1-28, 1955.

MADDISON, A. Quantifying and interpreting world development: macromeasurement before and after Colin Clark. Australian Economic History Review, v. 44, n. 1, p. 1-34, 2004. 
MADDISON, A. Statistics on world population, GDP and per capita GDP, 1-2008 AD. 2010. Disponível em: <http://www.ggdc.net/maddison/Historical_Statistics/horizontal-file_02-2010. xls >. Acesso em: 31 mar. 2010.

MAIA, A. G. A contribuição das fontes de rendimento na dinâmica da distribuição espacial de renda no Brasil. Nova Economia, v. 20, n. 3, p. 461-490, 2010.

MILANOVIC, B. An estimate of average income and inequality in Byzantium around year 1000. Review of Income and Wealth, v. 52, n. 3, p. 449-470, Sept. 2006.

MILANOVIC, B.; LINDERT, P. H.; WILLIAMSON, J. G. Pre-industrial Inequality. The Economic Journal, v. 121, n. 551, p. 252-272, Mar. 2011.

MOSSI, M. et al. Growth dynamics and space in Brazil. International Regional Science Review, 26, n. 3, p. 393-418, 2003.

MYRDAL, G. Economic theory and under-developed regions. London: Methuen, 1963.

REIS, E. J. Income per capita of Brazilian municipalities in the 1870s. 2009. Disponível em: $<$ http://www.nemesis.org.br/sec-din5.php?id=0000000166Eji=pt $>$. Acesso em: 31 mar. 2010.

REIS, E. J.; PIMENTEL, M.; ALVARENGA, A. Áreas mínimas comparáveis para os períodos intercensitários de 1872 a 2000. 2007. Disponível em: <http://nemesis.org.br/sec-din5. php?id=0000000188Ei $=$ en $>$. Acesso em: 31 mar. 2010.

REIS, E.; MONASTERIO, L. M. Mudanças na concentração espacial das ocupações nas atividades manufatureiras do Brasil, 1872-1920. In: BOTELHO, T. R.; LEEUWEN, M. H. D. (Org.). Desigualdade social na América do Sul: perspectivas históricas. Belo Horizonte: Veredas e Cenários, 2010. p. 243-274.

RESENDE, G. M. Multiple dimensions of regional economic growth: the Brazilian case, 1991 -2000. Papers in Regional Science, v. 90, n. 3, p. 629-662, Aug. 2011.

ROSTOW, W. W. The stages of economic growth. The Economic History Review, v. 12, n. 1, p. 1-16, 1959.

WILLIAMSON, J. G. Regional inequality and the process of national development: a description of the patterns. Economic Development and Cultural Change, v. 13, n. 4, p. 1-84, July 1965. 


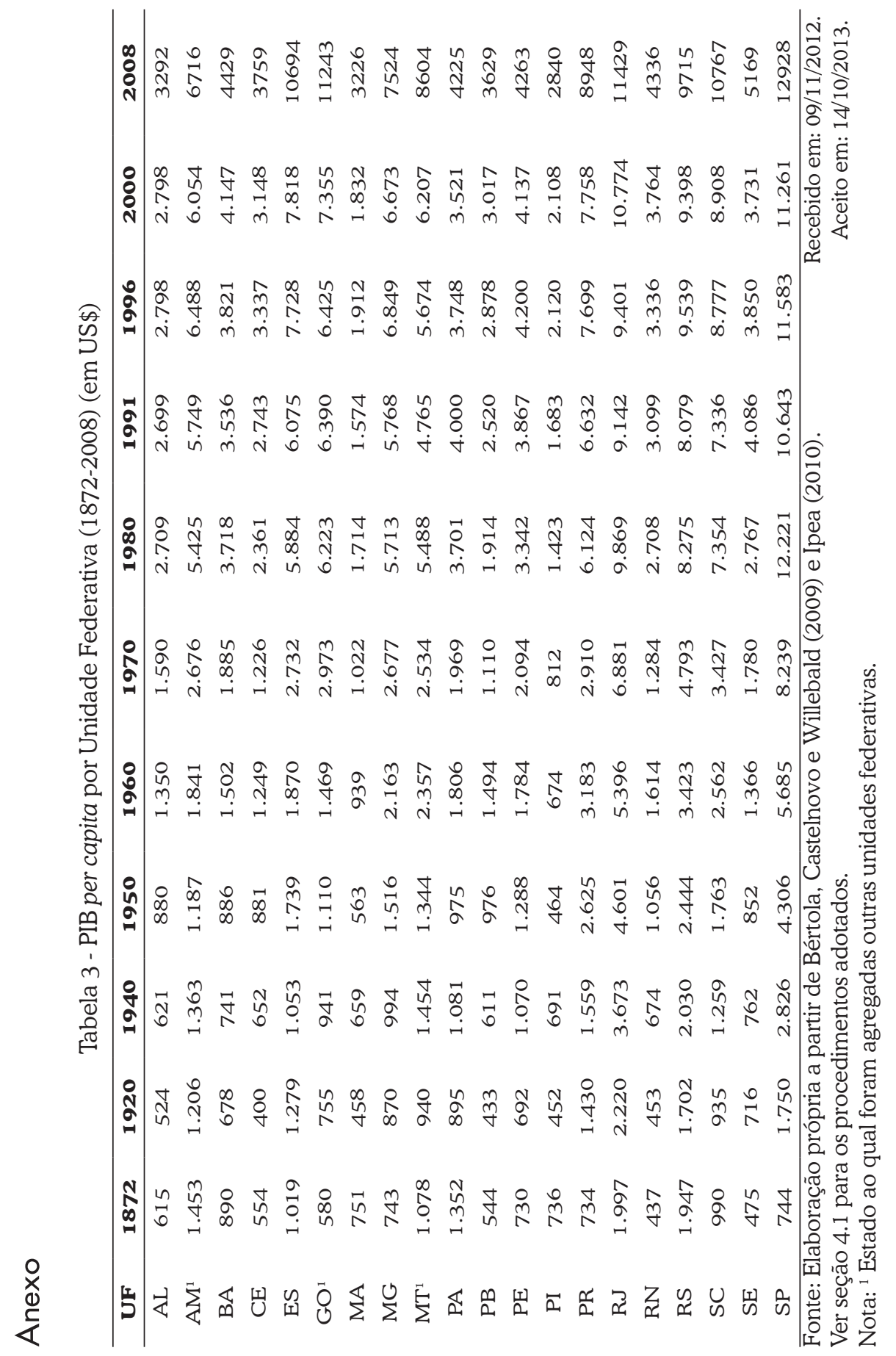

